\section{Declaración de conflictos de intereses: contribución a la mejoría en la calidad ética de las publicaciones}

\author{
MAURICIO SOTO SUBIABRE
}

\section{Conflict of interest disclosure as a tool to improve the reliability of research}

Conflicts of interest are situations in which professional judgment concerning a primary interest, tends to be influenced by a secondary interest. These conflicts could compromise the reliability of biomedical research and result in the dissemination of biased results. Therefore, it is essential to protect the integrity of them, because this information could be used in the development of health policy, medical education and clinical decision making. Conflicts of interest disclosure, is a bioethical tool that contributes to transparent these conflicts, but it is used inappropriately and insufficiently. To be useful, it must be based on clear principles, it should help to perform a critical analysis and should be considered in the design of every research project. This review is an analysis of the conflicts of interest disclosure, its scope and limitations and should contribute to develop a greater awareness of its importance.

(Rev Med Chile 2016; 144: 1067-1072)

Key words: Bioethics; Conflict of Interest; Disclosure.
Programa de Estudios Médicos

Humanísticos. Pontificia

Universidad Católica de Chile.

Recibido el 6 de agosto de 2015 , aceptado el 11 de junio de 2016 .

Correspondencia a: Mauricio Soto Subiabre Lira 61, Piso 2. Santiago. masoto5@uc.cl
L os conflictos de interés (CI) en la investigación biomédica potencialmente producen incompatibilidad de intereses, compromiso al juicio profesional en la actividad clínica y la toma de decisiones de los editores para decidir la publicación de un artículo ${ }^{1}$. Estos existen cuando se tiene la obligación moral de actuar en nombre de otros y esta acción se compromete por vínculos con un tercero, teniendo una multiplicidad de manifestaciones y no siempre con un desenlace incorrecto desde la ética, pero obligando que su manejo sea riguroso ${ }^{2}$. Cobran relevancia al comprometer el interés primario de una investigación, que es generar resultados válidos y confiables. Será fundamental promover y proteger la integridad de ellos en una investigación biomédica, dado su uso en políticas de salud, educación médica o toma de decisiones clínicas ${ }^{3}$.

Respecto del médico, clínico y/o investigador, los potenciales CI suelen darse relacionados con la industria comercializadora de productos médicos, sea por financiamiento de investigaciones o situaciones cotidianas (regalos, asesorías, pagos por conferencias, invitaciones a congresos o actividades de educación médica).

El análisis crítico de cualquier trabajo científico deberá objetivarse en su calidad metodológica y ética, involucrando aspectos tradicionales en ética de la investigación (revisión y aprobación por comité de ética de investigación, balance riesgo/ beneficio, consentimiento informado), como también presencia de $\mathrm{CI}$ de autores, instituciones financiadoras, editores y revista que publicará el artículo, que podrían sesgar los resultados ${ }^{4}$.

Los CI son de difícil control, sea por desconocimiento o por no identificarlos. La declaración de conflictos de intereses (DCI) es una herramienta que transparenta potenciales $\mathrm{CI}$, enmarcándose en un conjunto definido de buenas prácticas que mejora la calidad de una investigación ${ }^{5}$. 
La presente revisión, analizará el impacto de los CI en investigaciones biomédicas y reflexionará sobre la DCI, sus alcances y limitaciones, como una herramienta necesaria de protección de toda investigación.

\section{Conflicto de interés: definición}

Un CI corresponde a un grupo de condiciones en donde el juicio profesional acerca del interés primario, estaría influenciado por un interés secundario, produciendo incompatibilidad de intereses y compromiso al juicio profesional ${ }^{1,6,7}$. Se distinguen CI real, aparente y potencial ${ }^{8}$. Un CI real es un inaceptable conflicto de hecho entre los intereses del investigador, revisores o miembros del comité editorial y sus obligaciones con el interés primario de la investigación. Será $a p a-$ rente, cuando el interés personal no influye en el investigador, revisor o comité editorial, pero daría lugar a que otros consideren que influiría en el cumplimiento del interés primario. Será potencial cuando un interés privado pueda convertirse en un CI real.

Existen tres condiciones propias a los CI. Primero, es una situación objetiva que trae consigo un riesgo a la transparencia del proceso de publicación y difusión de una investigación ${ }^{9}$. Segundo, es independiente de la calidad moral de los involucrados. Tercero, no es un dilema ético ya que no hay competencia por prioridad de valores, dado que en un CI el interés primario siempre debe prevalecer ${ }^{10}$. Intereses secundarios son situaciones que pueden convertirse en conflictivas para un investigador o sociedad científica (Tabla 1).
Frente a un CI es dable preguntarse ¿qué es lo inmoral en ese juego de intereses? Un potencial CI no es inmoral en sí mismo, ya que es una situación inherente al reunirse intereses divergentes de personas y porque alude a una condición y no una conducta ${ }^{5}$. Puede que nunca se enmarque dentro la inmoralidad, sea por la rectitud moral o falta de ocasión, asumiendo así su potencialidad como resultado de la acción de un sujeto, quien se propone un fin central e inmediato. Es fundamental preguntar ¿quién tiene el conflicto de interés, qué involucra ese conflicto y por qué se llegó a ello?", permitiendo identificar el agente, el tipo de acción, el fin inmediato y las intenciones últimas, lográndose un juicio moral prudencial de la acción ${ }^{14}$.

Por tanto, para lograr una diferenciación entre la potencialidad de un CI (condición) y uno real (hecho), es necesario entenderlo desde la funcionalidad de una persona que ejecuta una acción determinada y una intención ordenada a un fin.

\section{Conflictos de intereses e impacto en la investigación biomédica}

Los CI disminuyen la confianza de los lectores y confieren un error (no metodológico) adicional a la investigación. Un error no metodológico es el sesgo de publicación o la tendencia a publicar resultados estadísticamente significativos. Está demostrado que revisores y comités editoriales favorecen estudios con resultados positivos o en ocasiones los financiadores llegan a involucrarse en el diseño, recolección de datos, análisis y redacción

Tabla 1. Categorización de los intereses secundarios ${ }^{11-13}$

\begin{tabular}{|ll|}
\hline Categoría de interés secundario & Elementos \\
\hline Financiamiento directo & $\begin{array}{l}\text { Pagos por conferencias, escribir artículos, reclutar pacientes para estudios clínicos } \\
\text { o por investigar un determinado producto }\end{array}$ \\
\hline Financiamiento indirecto & Regalos, viajes, comidas \\
\hline Generados por el médico & $\begin{array}{l}\text { Negocios personales, recibir comisiones por derivación de pacientes, solicitud de } \\
\text { exámenes o extender recetas }\end{array}$ \\
\hline Desarrollo laboral y/o académico & Conseguir prestigio, reconocimiento académico o ascenso \\
\hline Otros & $\begin{array}{l}\text { Médico clínico es a su vez investigador financiado de un producto; cuando un } \\
\text { médico no utiliza los recursos necesarios y disponibles y con ello disminuye los } \\
\text { costos en el sistema de salud; realizar consultarías para respaldar estudios o } \\
\text { políticas de mercado }\end{array}$ \\
\hline
\end{tabular}


final del manuscrito ${ }^{15}$. Otro error no metodológico es reportar resultados sesgados, al seleccionarlos o manipularlos para su publicación, motivados por un interés secundario que se desea privilegiar. Los CI predisponen a los autores a obtener resultados favorables o estadísticamente significativos para un financiador, revisiones incompletas por parte de pares o parcialidad al decidir la publicación.

Se hace necesaria una mejoría permanente en las capacidades analíticas de un trabajo, para asegurar la precisión de una investigación, no sólo en su originalidad, validez científica e impacto, sino también con énfasis en los sesgos no metodológicos ${ }^{16}$.

Los CI de los investigadores impactan también en la decisión de las personas de participar como sujetos de investigación. Una revisión sistemática, incluyendo artículos entre 1950 y 2010, mostró que $48 \%$ de ellos daba importancia a conocer los CI de autor(es), principalmente financiero ${ }^{17}$. Otra investigación cualitativa, por la técnica de grupos focales, reveló que los potenciales participantes deseaban conocer acerca de los CI de investigadores, no reconociendo si ello afectaría su participación. Respecto del financiamiento, algunos deseaban conocer esa información detallada, pero otros asumían el riesgo de participar, independiente de montos y origen ${ }^{18}$.

Por tanto, autores, revisores y comité editorial asumen un doble rol: reconocer los sesgos en su investigación y reportar y manejar potenciales $\mathrm{CI}^{19}$.

\section{Manejo del conflicto de intereses en investigaciones científicas}

Una revisión sistemática de trabajos originales entre 1980 y 2002 intentando responder la pregunta ¿cuán comunes son las relaciones financieras entre la industria, investigadores e instituciones académicas? Encontraron que un cuarto de los investigadores en biomedicina recibieron financiamiento de la industria, $43 \%$ recibió regalos relacionados con el tema de investigación y un tercio tenía relaciones financieras con la industria, independiente si los investigadores pertenecían a una institución académica ${ }^{20}$.

Dado que los CI pueden afectar la integridad de una investigación, se exige declararlos y vigilarlos, siendo la solución más utilizada la
DCI. En general, esta debe ser una normativa del comité editorial, originada inexcusablemente en los autores, contener información completa que proporcione un beneficio real para analizar un trabajo y reconocerla como una forma más en el manejo de un CI. Los contenidos mínimos exigibles serían: potenciales y actuales intereses económicos, participación financiera con la industria y percepción de honorarios ${ }^{10}$. Se ha reportado que $93 \%$ de revistas médicas en inglés tienen políticas de DCI para los autores, en comparación con $46 \%$ y $40 \%$ para los revisores y editores, respectivamente. En cuanto a la publicación de la DCI, este mismo estudio mostró que $57 \%$ publica las DCI de autores, $3 \%$ de revisores y $12 \%$ de editores ${ }^{21}$.

Sin embargo, ¿dónde se ubica la DCI, en el contexto de la ética de investigación? Constituye un punto de referencia, libremente aceptado por científicos e instituciones y enmarcado en un conjunto de buenas prácticas ${ }^{5}$. Cabe reflexionar, si la DCI es una estrategia de mitigación de los CI o si puede tener un efecto negativo, toda vez que sea el simple cambio de un sesgo secreto a uno conocido, con un contenido centrado en información financiera, desconociendo otras condiciones configuradas como potenciales CI. Lo más complejo sería que la DCI sea percibida como una forma de no asumir la responsabilidad en el manejo de los $\mathrm{CI}^{22}$.

\section{Una declaración de conflictos de intereses útil}

Es fundamental reconocer el impacto de los CI en el desarrollo y divulgación de una investigación. Son factores relevantes: comprender su definición y clasificación, impacto en la evaluación crítica de la investigación, responsables de reconocer el conflicto y darlo a conocer, normas reguladoras de sociedades científicas o comités editoriales y la aplicabilidad de la información contenida en la DCI.

Los principales elementos en la configuración de una DCI útil son: origen (autor o comité editorial) formato y contenido (conexiones financieras, propiedad intelectual, pertenencia a comité editorial de una revista, por ejemplo). Clarificando estos puntos, la DCI proporcionaría información real, específica, completa y necesariamente individualizada para cada participante. 
Existen situaciones potencialmente conflictivas en una DCI que mermarían su utilidad. Primero, centrar toda la DCI en lo financiero; segundo, no declarar pertenencia de los autores al comité editorial de la revista donde solicitan publicar; y tercero, no declarar la representación de las instituciones (universidades, sociedades científicas, por ejemplo) en el comité editorial a través de sus miembros. Esta información, si bien se puede discutir su impacto, constituye un potencial o aparente CI y por tanto, debe ser visibilizada, ya que potencia la sensibilización necesaria para lograr una diferenciación entre CI real, aparente o potencial. El comité editorial debe ser el primero en responsabilizarse en evidenciar lo anterior, solicitar una DCI completa y rechazar un trabajo hasta que lo anterior se cumpla. De no lograrlo, reflejaría normativas flexibles y una DCI ineficiente, no permitiendo un análisis crítico de los trabajos y una falta de sensibilidad del comité editorial en detectar potenciales $\mathrm{CI}^{23}$.

Desconocer lo anterior, haría que el contenido de la DCI sea mínimo, transparentando parcialmente la investigación y configurándose como un acto de buena práctica y de alineación con normativas internacionales, no resultantes de procesos reflexivos individuales (autores, revistas o sociedades científicas). La peor situación es una DCI incompleta, limitada a lo financiero, perdiendo utilidad y credibilidad. Por lo anterior, es importante dar una mirada a normativas internacionales.

El Comité Internacional de Editores de Revistas Médicas (ICMJE) considera que las etapas de un trabajo son: escritura, revisión por pares y toma de decisiones del comité editorial. Lo fundamental es la independencia en el manejo de los datos, evitando manipulaciones a la inspiración inicial del trabajo y reconocer que la DCI debe involucrar autores y también, a revisores y comité editorial, permitiendo que la evolución de la investigación sea concordante con sus méritos. Así, revisores y editores adquieren una responsabilidad intrínseca promoviendo la declaración de sus $\mathrm{CI}$ y exigiendo una DCI real y completa. Estas recomendaciones proporcionan ayuda para autores, editores y revisores, cuya finalidad es la publicación de artículos confiables, claros e imparciales ${ }^{24}$.

El ICMJE, publicó en 2013 recomendaciones dirigidas a revisar las prácticas y los estándares éticos en el desarrollo y publicación de investiga- ciones en revistas médicas. Estas recomendaciones se agrupan en tres áreas: descripción de roles y responsabilidades de autores, editores, revisores; cuestiones acerca de la publicación del trabajo y preparación del manuscrito y revisión.

Acciones concretas de los autores serán revelar financiamiento o conexiones financieras con el tema investigado. Revisores deben revelar cualquier situación que comprometa sus opiniones o interpretación del manuscrito, excusarse de revisar un trabajo si es necesario y no usar la información contenida en él para fines personales. Editores no deben tener compromisos financieros relativos a las materias juzgadas, marginarse si es necesario, exigir la DCI y no aprovechar personalmente la información ${ }^{24}$.

En el 2009, un trabajo que evaluó las instrucciones a los autores de 256 revistas médicas de alto impacto, encontró que $89 \%$ presentaba políticas relativas a $\mathrm{CI}, 77 \%$ proporcionaba definiciones del tema y $57 \%$ solicitaba a los autores firmar una $\mathrm{DCI}^{25}$. La evolución en el tiempo de las políticas de CI en revistas científicas es evidente, ya que en 1997 sólo 16\% de 1.396 revistas (en todas las disciplinas científicas) tenían políticas sobre DCI y el 2008 alcanzó $89 \%{ }^{25,26}$. Respecto de los reglamentos de publicación, en el 2004 33\% de las revistas publicaban on line sus políticas sobre la DCI, a pesar de que $80 \%$ reportó tenerlas ${ }^{27}$. A nivel nacional, en el 2005, un análisis retrospectivo mostró que $42 \%$ de los artículos de investigación publicados en la Revista Médica de Chile explicitó sus fuentes de financiamiento ${ }^{28}$.

Existe un aumento en la demanda de una DCI financieros por parte de pacientes, participantes en investigaciones y lectores. Una revisión sistemática encontró que los pacientes, en relación a los conflictos financieros, creen que condicionan la conducta profesional y debiesen declararse. Pacientes, médicos y participantes en investigaciones creen que estos conflictos disminuyen la calidad de la investigación y los CI financieros afectarían el bienestar de los participantes en una investigación ${ }^{29}$.

La DCI es una estrategia válida para manejar y mitigar los CI, pero podría tener un efecto negativo, al construirse desde la simple intención de cumplir una tendencia voluntaria y no regulada, llevando al statu quo y no potenciando una DCI bien fundamentada y centrada en todos los involucrados en la investigación. 


\section{La declaración de conflictos de intereses desde la bioética}

Todo debate respecto de la DCI es un tema de la bioética propiamente tal, ya que se necesita armonizar el interés básico de una investigación con intereses secundarios. En el 2013, la declaración de Helsinki de la Asociación Médica Mundial, reafirmó el tema de los CI haciendo referencia a que "publicaciones deben citar fuentes de financiamiento, afiliaciones institucionales y conflictos de intereses. Los informes sobre investigaciones que no se ciñan a los principios descritos en esta Declaración no deben ser aceptados para su publicación". Pero, como plantea Lolas, "la real protección de los sujetos y los principios éticos residen en la conciencia de los investigadores", adquiriendo relevancia los imperativos de la investigación. Estos incluyen: el mérito técnico o metodológico; mérito científico promoviendo la reflexión en una disciplina y aporte al conocimiento; $y$ finalmente, un mérito social o legitimidad de la investigación ${ }^{29}$. Es esto último lo que debe incentivarse en investigadores a través de la adquisición de un conocimiento claro y comprensible del origen de los CI, clasificación, impacto y manejo.

Las políticas de comités editoriales, deben exigir y fundamentar la obligatoriedad de una DCI, reflejándose en reglamentos de publicación claros y de interpretación unívoca con los fines de una investigación. Los autores, revisores y miembros del comité editorial, deberán asumir su responsabilidad respecto de la información entregada en la declaración, ser corroborable y que un comité editorial pueda rechazar su artículo en la medida que considere una DCI incompleta.

Finalmente, una DCI debe constituirse en un requisito obligatorio para el diseño, escritura y publicación de una investigación. Será obligatorio que las revistas médicas asuman su responsabilidad con una cultura científica sensibilizada en promover investigación centrada en su interés primario. La DCI se debe anticipar a una condición potencial de CI, pero debe ser reconocida como una herramienta falible y que el simple acto de declarar no es suficiente. De esta manera, se perderá el miedo a enfrentarse a la DCI y se comenzará a reconocer que ciertas actividades $o$ vinculaciones con la industria, pueden afectar el juicio profesional y deben ser de conocimiento público.

\section{Referencias}

1. Ortiz A. Gestión Clínica y Conflictos de Intereses. Acta Bioeth 2009; 15 (2): 157-64.

2. La Rosa E. Los conflictos de intereses. Acta Bioeth 2011; 17 (1): 47-54.

3. Buerba R, Fu M, Grauer J. Discrepancies in spine surgeon conflict of interest disclosures between a national meeting and physician payment listings on device manufacturer web sites. Spine 2013. http://dx.doi.org/10.1016/j.spinee.2013.05.032

4. Morreim H. The Devil in the Detail (ing). Am J Bioeth 2010; 10 (1): 15-7.

5. Thompson D. Understanding financial conflicts of interest. New Engl J Med 1993; 329: 573-6.

6. Lama A. El médico y los conflictos de interés. Rev Med Chile 2003; 131: 1463-8.

7. Organisation for Economic Co-Operation and Development. Guía sobre el Manejo de Conflictos de Intereses en el Sector Público y Experiencias Internacionales 2003. (Consultado el 12 de diciembre de 2015).

8. Zalaquett J. Conflictos de intereses: normas y conceptos. Anuario de Derechos Humanos 2011; (7): 179-189. doi:10.5354/0718-2279.2011.17363.

9. Camí J. Conflicto de intereses e investigación clínica. Med Clin-Barcelona 2013; 105: 174-9.

10. Kaliski S. Conflict of interest: The elephant in your practice. Afr J Psychiatry 2013; 16: 161-5.

11. Lolas F, Rodríguez E. Conflictos de interés en la investigación bioética y psicosocial. En Fernando Lolas, Álvaro Quezada y Eduardo Rodríguez (Editores), Investigación en Salud. Dimensión Ética. Santiago, Chile: Ediciones Universidad de Chile 2006. p. 231-8.

12. Steinbrook R. Controlling conflict of interest-Proposals from the Institute of Medicine. New Engl J Med 2009; 360 (21): 2160-3.

13. Miller D. Disclosure of conflicts of interest in biomedical publications. Can J Anaesth 2008; 55 (5): 265-9.

14. Gómez-Lobo A. Los Bienes Humanos. Ética de La Ley Natural. Santiago: Editorial Mediterráneo 2006.

15. Odierna D, ForsythS, White J, Bero L. The Cycle of Bias in Health Research: A Framework and Toolbox for Critical Appraisal Training. Accountability in Research 2013; 20 (2): 127-41.

16. Kirkby H, Calvert M, Draper H, Keeley T, Wilson S. What potential research participants want to know about research: a systematic review. Brit Med J 2012; (2). doi:10.1136/bmjopen-2011-000509.

17. Weinfurt K, Friedman J, AIIsbrook J, Haft M, Sugarman J. Views of Potential Research Participants on Financial Conflicts of Interest Barriers and Opportunities for 
Effective Disclosure. J Gen Intern Med 2006; 21: 901-6.

18. Warner T, Gluck J. What do we really know about conflicts of interest in biomedical research? Psychopharmacology 2003; 171: 36-46.

19. Bekelman J, Li Yan, Gross G. Scope and impact of financial conflicts of interest in biomedical research: sistematic review. J Amer Med Assoc 2003; 289: 454-65.

20. Cooper R, Gupta M, Wilkes, M. Conflict of Interest Disclosure Policies and Practices in Peer-reviewed Biomedical Journals. J Gen Intern Med 2006; 21: 1248-52.

21. The PLoS Medicine Editors. Does Conflict of Interest Disclosure Worsen Bias? PLoS Med 2012; 9 (4): e1001210. doi:10.1371/journal.pmed.1001210.

22. International Committee Medical Journals Editors. Recommendations for the Conduct, Reporting, Editing, and Publication of Scholarly Work in Medical Journals. 2013. Recuperado el 04 de junio de 2014, en http://www. icmje.org/icmje-recommendations.pdf.

23. Soto M. Declaración de conflictos de intereses en investigaciones publicadas en dos revistas científicas nacionales, entre los años 2002 y 2012 (tesis de magíster). Santiago: Pontificia Universidad Católica de Chile; 2014.
24. Blum J, Freeman K, Dart D, Cooper R. Requirements and Definitions in Conflict of Interest Policies of Medical Journals. J Amer Med Assoc 2001; 302: 20.

25. Krimsky S, Rothenberg LS. Conflict of interest policies in science and medical journals: editorial practices and author disclosures. Sci Eng Ethics 2001; 7 (2): 205-18.

26. Ancker JS, Flanagin A. A comparison of conflict of interest policies at peer-reviewed journals in different scientific disciplines. Sci Eng Ethics 2007; 13 (2): 147-57.

27. Licurse A, Barbe E, Joffe S, Gross C. The Impact of Disclosing Financial Ties in Research and Clinical Care A Systematic Review. Arch Intern Med 2010; 170 (8): 675-82.

28. Romero W, Salas S. ¿Explicitan los autores de la Revista Médica de Chile sus fuentes de financiamiento? Rev Med Chile 2007; 135: 473-9.

29. Lolas F. Aspectos Éticos de la Investigación Biomédica Conceptos Frecuentes En Las Normas Escritas. En Fernando Lolas y Álvaro Quezada (Editores), Pautas éticas de investigación en sujetos humanos: Nuevas Perspectivas. Santiago, Chile: Programa Regional de Bioética OPS/OMS; 2003. p. 65-70. 